\title{
EFICACIA Y SEGURIDAD DE CLONIDINA VERSUS PLACEBO PARA ANSIEDAD EN ODONTOLOGÍA
}

\author{
Patricia Bermúdez-Reyes ${ }^{\star}$, M.Sc. ${ }_{1}$, Ana Karina Támara-Eraso, M.Sc. ${ }_{{ }_{2}}$ Jorge William Vargas, M.Sc.
}

\author{
${ }_{1}$ Universidad Cooperativa de Colombia, Facultad de Odontología, sede Envigado, Colombia \\ ${ }_{2}$ Médica de la Universidad de Cartagena y Médica de la Fundación Médico Preventiva, Colombia
}

Recibido: 20 de junio del 2013. Aprobado: 23 de julio del 2013.

*Autor de correspondencia: Patricia Bermúdez Reyes, Facultad de Odontología, Universidad Cooperativa de Colombia, Medellín, Colombia, Calle 48C sur n. ${ }^{4}$ 42D-47 Int. 237, Medellín, Colombia, correo electrónico: patricia.bermudez@campusucc.edu.co

Cómo citar este artículo: Bermúdez-Reyes P, Támara-Eraso AK, Vargas JW. Eficacia y seguridad de clonidina versus placebo para ansiedad en odontología. Rev Nac Odontol. 2013; 9(17): 7-15.

Resumen. Introducción: para el control de la ansiedad en la atención odontológica existen diferentes estrategias. El objetivo de este trabajo fue determinar la eficacia y la seguridad de la medicación con clonidina versus placebo, para disminuir la ansiedad y el dolor en la extracción de terceros molares. Métodos: ensayo clínico, placebo controlado, aleatorizado, triple ciego y diseño de grupos paralelos; participaron 40 pacientes sanos con edades entre 14 y 40 años, que serían sometidos a cirugía de terceros molares, con puntuación $\geq 13$, según la escala de ansiedad dental modificada (MDAs); 20 pacientes participaron por grupo y recibieron clonidina de 150 mcg, al control placebo. Se aleatorizaron por bloques balanceados y el enmascaramiento consistió en un tableteado uniforme. Desenlace primario: cambio en la ansiedad, según MDAs; secundario: signos vitales, cambio en la escala del dolor y efectos secundarios. Resultados: la ansiedad disminuyó en ambos grupos, sin diferencias significativas entre el grupo clonidina y el grupo placebo $(\mathrm{p}=0,704)$; en el grupo experimental disminuyó la presión media al compararla con la del grupo placebo $(\mathrm{p}=0,033)$. Conclusión: la clonidina es segura, y aunque al compararla con el placebo no mostró diferencias significativas en disminución de la ansiedad, en el grupo que la recibió se observó un descenso en la presión arterial media, lo que puede reflejar menor ansiedad, si consideramos sus signos somáticos.

Palabras clave: ansiedad, clonidina, extracción del tercer molar, Propanolol.

Efficacy and Safety of Clonidine versus Placebo for Anxiety in Odontology

Abstract. Introduction: There are different strategies for anxiety control in dentistry. The objective of this study was to determine the efficacy and safety of clonidine medication versus placebo to decrease anxiety and pain in third-molar extraction. Methods: a placebo controlled clinical trial, randomized, triple-blind, parallel group design, included 40 healthy patients aged 14 to 40 , who would be undergoing third molar surgery, with a score $\geq 13$, in accordance with the Modified Dental Anxiety Scale (MDAS); there were 20 patients per group who received 150 mcg clonidine and a control group that received the placebo. The groups were randomized in balanced blocks, and the masking consisted of uniform tableting. Primary outcome: change in anxiety according to MDAS; secondary outcome: vital signs, change in the pain scale and side effects. Results: decreased anxiety in both groups, with no significant differences between the clonidine group compared with placebo $(\mathrm{p}=0.704)$; in the experimental group average pressure decreased when compared with the placebo $(\mathrm{p}=0.033)$. Conclusion: clonidine is safe, and even though when compared to the placebo showed no significant anxiety reduction, in the clonidine group a decrease in average arterial pressure was observed, which could reflect reduced anxiety, considering its somatic signs.

Keywords: anxiety, clonidine, third molar extraction, Propranolol.
Eficácia e segurança de clonidina versus
placebo para ansiedade em odontologia

Resumo. Introdução: para o controle da ansiedade no atendimento odontológico, existem diferentes estratégias. O objetivo deste trabalho foi determinar a eficácia e a segurança do medicamento com clonidina versus placebo, para diminuir a ansiedade e a dor na extração de terceiros molares. Métodos: ensaio clínico, placebo controlado, aleatorizado, triplo-cego e desenho de grupos paralelos; participaram 40 pacientes saudáveis com idade entre 14 e 40 anos, que seriam submetidos à cirurgia de terceiros molares, com pontuação $\geq 13$, segundo a escala de ansiedade dental modificada (MDAS); 20 pacientes participaram por grupo e receberam clonidina de $150 \mathrm{mcg}$, ao controle placebo. Aleatorizaram-se por blocos balanceados e o mascaramento consistiu em um tableteado uniforme. Desenlace primário: mudança na ansiedade, segundo MDAs; secundário: sinais vitais, mudança na escala da dor e efeitos secundários. Resultados: a ansiedade diminuiu em ambos os grupos, sem diferenças significativas entre o grupo clonidina e o grupo placebo $(p=0,704)$; no grupo experimental diminuiu a pressão média ao compará-la com a do grupo placebo $(\mathrm{p}=0,033)$. Conclusão: a clonidina é segura e, embora ao compará-la com o placebo não tenha mostrado diferenças significativas na diminuição da ansiedade, no grupo que a recebeu, observou-se um descenso na pressão arterial média, o que poderia refletir uma menor ansiedade, se considerássemos seus sinais somáticos.

Palavras-chave: ansiedade, clonidina, extração do terceiro molar, Propanolol. 


\section{Introducción}

En muchos países se ha reconocido que la ansiedad que produce la atención odontológica es un importante problema de salud. Una gran proporción de la población de Estados Unidos, Reino Unido, Holanda, Suiza, Dinamarca, Noruega, Hong Kong y Canadá ha reportado algún grado de ansiedad relacionado con la visita y el tratamiento odontológico [1]. En Bogotá, Colombia, en el 2009, Caycedo y sus colaboradores encontraron en el estudio Ansiedad al tratamiento odontológico: características y diferencias de género, una prevalencia de ansiedad dental de 47,2\% en aquellos procedimientos que incluyen jeringas, una del $34,7 \%$ ante la anticipación de la extracción de una pieza dental y una del $25,4 \%$ ante la anticipación del uso de la fresa dental [2].

Para el control de la ansiedad en el consultorio odontológico se han utilizado diferentes estrategias farmacológicas y no farmacológicas; sin embargo, es importante destacar que en un gran porcentaje de los casos no se implementa ninguna medida para atenuarla. En el campo de las estrategias no farmacológicas se encuentran: relajación, aromaterapia, hipnosis, musicoterapia, visualización de videos y terapia cognitiva de desensibilización, entre otras [3-5]. En cuanto a las terapias farmacológicas se han utilizado técnicas de sedación consciente con óxido nitroso, Midazolam, Propofol y muchos otros, así como también antihistamínicos, inductores del sueño $[6,7]$ y antihipertensivos, considerados estos últimos como ansiolíticos de segunda línea, debido a que este efecto se presenta como evento secundario.

Sin embargo, una de las limitaciones de las terapias farmacológicas, en el caso de la sedación, es que se debe realizar en un ámbito hospitalario bajo la supervisión de un anestesiólogo, lo que incrementa el costo para el paciente y también aumenta la posibilidad de efectos secundarios indeseables.

Por otro lado, se ha demostrado que en los pacientes muy ansiosos el acto quirúrgico se prolonga y además refieren más dolor postoperatorio con una recuperación más complicada [8-10]. Con el fin de buscar una alternativa farmacológica segura, con mínimos efectos secundarios y que permitiera un manejo ambulatorio en el consultorio odontológico, se decidió realizar el presente estudio. El objetivo fue determinar la eficacia y la seguridad de la Clonidina para manejar la ansiedad transitoria y el dolor intra y postoperatorio en pacientes sometidos a cirugía ambulatoria de terceros molares. La clonidina ha mostrado su eficacia y seguridad tanto en adultos como en niños, en los diferentes contextos en los que ha sido estudiada $[11,12]$.

\section{Métodos}

Se realizó un ensayo clínico controlado, aleatorizado, triple ciego, con un diseño de grupos paralelos. La población estuvo conformada por pacientes con edades entre 14 y 40 años, que serían sometidos a una cirugía electiva de los terceros molares; no se tuvo en cuenta el tipo de cirugía. Se incluyeron cirugías de terceros molares con vía abierta o en posición ectópica, realizadas por estudiantes de pregrado bajo la supervisión del docente de cirugía. Son pacientes normo-tensos y prehipertensos, según la directriz del viI Joint Nacional Committee, que presentaron ansiedad dental por encima de 13 puntos, de acuerdo con la escala MDAs, que pesaron entre 45 y 90 kilogramos, que voluntariamente aceptaron participar en el estudio y firmaron el consentimiento informado; en el caso de los menores de edad, el consentimiento fue firmado por su representante legal, contando con el asentimiento verbal del niño.

Se excluyeron aquellos pacientes que recibían alguna medicación por patologías de tipo crónico, a pacientes afroamericanos, por ser más susceptibles a presentar trastornos en la presión arterial, a los que tuvieran antecedentes de enfermedad cardiovascular o enfermedad sistémica crónica como: diabetes, hipertiroidismo o hipotiroidismo, síndrome de Addison, insuficiencia renal, hipertensión arterial o enfermedad hepática; a mujeres embarazadas o en período de lactancia y a pacientes con diagnóstico de trastorno de ansiedad o trastorno depresivo. Se integraron dos grupos: uno experimental, al que se le administró clonidina de 150 mcg, y un grupo de control, al que se le administró un placebo. Se contó con el aval del comité de ética de la Universidad Centro de Estudios para la Salud, CES. La asignación de los participantes a alguno de los grupos se realizó al azar, utilizando el método de aleatorización por bloques balanceados, a cargo de un asesor externo, y se utilizó un enmascaramiento con un tableteado uniforme, para garantizar el triple cegamiento.

Los medicamentos fueron administrados una hora antes del procedimiento quirúrgico. La escala MDAS se utilizó para valorar el grado de ansiedad de los pacientes, mediante el diligenciamiento de un cuestionario autoadministrado. Aquellos que presentaron niveles 
de ansiedad por encima de los 13 puntos se consideraron elegibles para ingresar al estudio, siempre y cuando cumplieran con los demás criterios de inclusión. Esta medición se realizó antes de administrarles la intervención. Al finalizar la cirugía se aplicó nuevamente la escala.

Para valorar el dolor percibido por el paciente durante el procedimiento y en el postoperatorio, se utilizó la escala numérica del dolor; se interrogó al paciente después del procedimiento, pidiéndole que indicara entre 0 y 10 , siendo 0 ningún dolor y 10 el máximo dolor; se midió nuevamente el dolor, vía telefónica, a las 6 y a las 24 horas después de la administración del tratamiento; se registraron los signos vitales (presión arterial, frecuencia cardíaca, frecuencia respiratoria y saturación de oxígeno) mediante un monitor multiparámetros (modelo trio básico marca Datascope), y los resultados se registraron en un formulario diseñado por los investigadores, de la siguiente forma: antes de la administración del medicamento, durante la cirugía —en tres ocasiones (al ser aplicada la anestesia local, durante la luxación del diente y al momento de suturar) - y al finalizar el procedimiento. El anestésico local que se utilizó fue la Prilocaína con Felipresina, para evitar interacciones con los dos medicamentos que se utilizaron. Al finalizar el procedimiento (aproximadamente a las tres horas de haber ingerido el medicamento), se registraron los efectos adversos de la intervención, con la interrogación personal del paciente, y a las $6 \mathrm{y}$ a las 12 horas se realizó vía telefónica.

Ante los procedimientos realizados a los pacientes al final de la mañana, por lo cual correspondía llamarlos por teléfono a altas horas de la noche, se les instruyó para que se comunicaran telefónicamente en caso de presentar algún evento adverso; a la mañana siguiente se realizó la medición correspondiente a las 24 horas. En el formulario también se consignaron las variables sociodemográficas: edad, sexo, estrato socioeconómico, tipo de afiliación al sistema de salud y peso; por otra parte, se describieron las características intraoperatorias: tiempo de duración del procedimiento, número de carpules administrados, número de dientes extraídos, cuáles dientes fueron extraídos (superiores o inferiores) y las complicaciones intraoperatorias.

El desenlace primario fue el cambio en la escala MDAS, antes y después de la administración de la intervención. Los desenlaces secundarios fueron: registro de los signos vitales (presión arterial, frecuencia cardíaca, frecuencia respiratoria, saturación de oxígeno), antes de la intervención, durante la cirugía y al finalizarla; cambios en la escala verbal numérica del dolor, después de la cirugía y en las 24 horas siguientes; por último, se realizó la frecuencia de efectos secundarios.

La base de datos se digitó en Excel Microsoft Office 2010; para el procesamiento de los datos recolectados se utilizó el programa estadístico spss 19. Inicialmente se llevó a cabo una exploración de la información por medio de gráficas y tablas de frecuencia. Para describir las variables cualitativas se utilizaron frecuencias absolutas y relativas, y para las variables cuantitativas se utilizó el promedio y su respectiva desviación estándar (DE) o la mediana y el rango intercuartílico (RIQ), según la distribución de los datos.

Para establecer la comparabilidad de los grupos se realizó un análisis de varianza, con las medidas de base de las variables cuantitativas, siempre y cuando las mismas presentaran una distribución normal; en caso contrario, se utilizó la prueba no paramétrica de Kruskall Wallis; para las variables cualitativas se utilizó la prueba $\mathrm{Chi}^{2}$, la variable respuesta principal y la media de la escala de ansiedad MDAS, comparada con el grupo control placebo mediante la prueba no paramétrica de Mann-Whitney.

Para comparar los signos vitales de los grupos y en los diferentes momentos evaluados se utilizó el Anova de dos vías con un factor relacionado. $Y$ en caso de encontrar diferencias se realizaron las comparaciones múltiples mediante la prueba de Bonferroni. Para evaluar la correlación entre la duración del procedimiento y el número de dientes extraídos, se calculó el coeficiente de correlación de Spearman. La significancia para todas las pruebas fue de 0,05 y se calcularon los intervalos de confianza al 95\% para cada una de las diferencias de medias entre grupos. La investigación se acogió a los principios de las buenas prácticas clínicas en investigación y se cumplió bajo los criterios de la guía ICH Guideline on Good Clinical Practice, originada en la declaración de Helsinki (ICH, Harmonised Tripartite Guideline).

\section{Resultados}

En la clínica de cirugía de la Facultad de Odontología de la Universidad Cooperativa de Colombia, en el período comprendido entre marzo del 2012 y abril del 2013 fueron operados 482 pacientes, de los cuales 60 fueron seleccionados según los criterios de elegibilidad $\mathrm{y}$ asignados aleatoriamente al grupo de tratamiento y al grupo de control placebo (figura 1). 


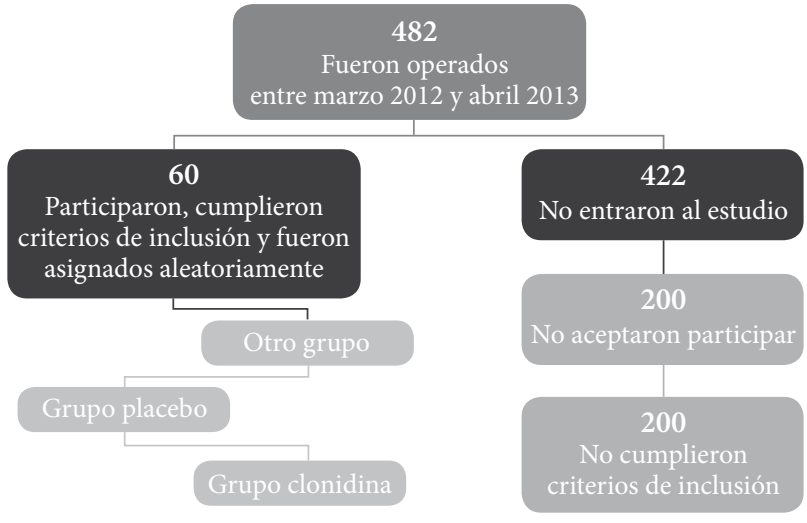

Figura 1. Flujograma de selección y exclusión de pacientes del estudio

Fuente: elaboración propia

La distribución por género en los grupos mostró mayor presencia de mujeres que de hombres, aunque sin diferencias significativas, con una proporción de las mujeres un poco mayor al $60 \%$ en los dos grupos; la edad y el peso promedio se presentaron de forma parecida en los dos grupos, oscilando entre 20-22 años y 59-62 kilos, respectivamente. La mayoría pertenecía al régimen subsidiado de salud y al estrato socioeconómico tres, con una distribución muy similar a la que se observa en la tabla 1.

No hubo pérdidas en el seguimiento, ya que fue un período corto. La mayoría de los desenlaces se evaluó después de la cirugía, pero para calificar la percepción del dolor y la presentación de los efectos secundarios se llevó a cabo una entrevista telefónica con cada uno de los participantes en las 24 horas siguientes al procedimiento quirúrgico y no se presentaron inconvenientes en la comunicación con los pacientes. El comportamiento de las variables que describen las características inherentes a los procedimientos quirúrgicos se muestra en la tabla 2.

En cuanto al número de dientes extraídos se observó una diferencia estadísticamente significativa entre los grupos evaluados. A la mayoría de los pacientes del grupo placebo, $16(80 \%)$, se les extrajo un solo diente, comparada con aquellos del grupo de Clonidina, 8 (40\%), $(\mathrm{p}=0,014)$; sin embargo, no hubo diferencia en cuanto al número de carpules de anestésico utilizados. Con respecto a las complicaciones durante la cirugía, se presentó dolor en los dos grupos en la misma proporción: cinco (25\%); dos pacientes (10\%) del grupo de Clonidina sufrieron hemorragias; no se presentaron casos de fractura de maxilar, daño del segundo molar u otros.
Tabla 1. Características basales de la población en estudio, de acuerdo con el grupo asignado

\begin{tabular}{|c|c|c|}
\hline Variables & Placebo & Clonidina \\
\hline \multicolumn{3}{|l|}{ Edad (años) ${ }^{* *}$} \\
\hline Media (DE) & $22,2(5,3)$ & $21,05(2,5)$ \\
\hline Rango & $14-35$ & $17-27$ \\
\hline \multicolumn{3}{|l|}{ Género $\mathrm{n}(\%)^{\star}$} \\
\hline Femenino & $12(60)$ & $13(65)$ \\
\hline Masculino & $8(40)$ & $7(35)$ \\
\hline \multicolumn{3}{|l|}{ Peso $(\mathrm{kg})^{\star *}$} \\
\hline Media (DE) & $63,2(10,6)$ & $64,23(77,7)$ \\
\hline \multicolumn{3}{|l|}{ Estrato socioeconómico $\mathrm{n}(\%)^{*}$} \\
\hline 1 & $3(15)$ & $1(5)$ \\
\hline 2 & $3(15)$ & $4(20)$ \\
\hline 3 & $12(60)$ & $14(70)$ \\
\hline 4 & $2(10)$ & 0 \\
\hline 5 & 0 & $1(5)$ \\
\hline 6 & 0 & 0 \\
\hline \multicolumn{3}{|l|}{ Afiliación al sistema de salud n\%* } \\
\hline Contributivo & $9(45)$ & $5(25)$ \\
\hline Subsidiado & $11(55)$ & $14(70)$ \\
\hline Vinculado & 0 & $1(5)$ \\
\hline Escala MDAS inicial. Media Ds $^{\star *}$ & $15,3(5,5)$ & $15,6(3)$ \\
\hline
\end{tabular}

${ }^{\star} \mathrm{Chi}^{2} \mathrm{p}>0,05{ }^{* *}$ Anova o Kruskall Wallis. $\mathrm{p}>0,05$

Fuente: original del estudio

Tabla 2. Distribución de las variables relacionadas con el procedimiento quirúrgico

\begin{tabular}{|c|c|c|c|c|}
\hline \multirow{2}{*}{ Variable } & & Placebo & Clonidina & \multirow{2}{*}{$\begin{array}{c}\text { Valor } \\
\mathrm{p}^{\star}\end{array}$} \\
\hline & & n (\%) & n (\%) & \\
\hline $\begin{array}{l}\text { Número } \\
\text { de dientes }\end{array}$ & Un diente & $16(80)$ & $8(40)$ & \multirow[t]{2}{*}{0,014} \\
\hline extraídos & Más de un diente & $4(20)$ & $12(60)$ & \\
\hline \multirow[t]{2}{*}{$\begin{array}{l}\text { Número } \\
\text { de carpules }\end{array}$} & Hasta 4 carpules & 1260 & $6(30)$ & \multirow{2}{*}{0,150} \\
\hline & Más de 4 carpules & $8(40)$ & $14(70)$ & \\
\hline Hemorragia & & 0 & $2(10)$ & 0,126 \\
\hline Dolor & & $5(25)$ & $5(25)$ & 1,000 \\
\hline
\end{tabular}

Fuente: elaboración propia

Al evaluar la duración del procedimiento entre placebo y clonidina, se encontró una diferencia significativa $(\mathrm{p}=0,026)$; sin embargo, no se encontró una correlación entre el número de dientes extraídos y el tiempo de la cirugía, coeficiente de correlación de Spearman $=0,1(p=0,539)$.

Cuando se compararon los cambios netos en la escala mDAs (antes y después), entre los grupos de la clonidina y el placebo no se observaron diferencias 
estadísticamente significativas (tabla 3). Es importante resaltar que en los dos grupos hubo disminución de la ansiedad.

Tabla 3. Análisis del cambio en la escala de ansiedad

\begin{tabular}{|l|l|l|l|}
\hline \multicolumn{4}{|c|}{ Dental MDAs en Clonidina vs placebo } \\
\hline Cambio MDAs & Mediana & Rango Intercuartil & Valor $\mathrm{p}^{*}$ \\
\hline Clonidina vs & 3,500 & $(0,000-6,750)$ & \multirow{2}{*}{0,704} \\
\hline placebo & 3,500 & $(0,250-4,750)$ & \\
\hline
\end{tabular}

${ }^{*}$ Mann-Whitney

$\mathrm{p}=<0,05$

Fuente: elaboración propia

La distribución de estos cambios netos en los valores de ansiedad se muestra en la figura 2 .



Figura 2. Cambio en la escala mDAs (antes y después) Fuente: elaboración propia

Como desenlace secundario se midieron los signos vitales; los valores promedios de la presión arterial en los distintos momentos evaluados se muestran en la tabla 4 para el placebo y la clonidina: el momento uno fue antes de administrar el medicamento; dos, durante la anestesia; tres, en la luxación u osteotomía; cuatro, en la sutura, y cinco, al finalizar la cirugía.
Al comparar la presión arterial entre el placebo y la clonidina se encontró una diferencia entre los grupos en la presión arterial media $(\mathrm{p}=0,033)$, no así en la presión sistólica ni diastólica; dentro de los grupos no hubo diferencia $(\mathrm{p}>0,05)$. En la figura 3 se observa cómo, mientras la tendencia del placebo es ascendente, la clonidina tiende a disminuir la presión arterial media.

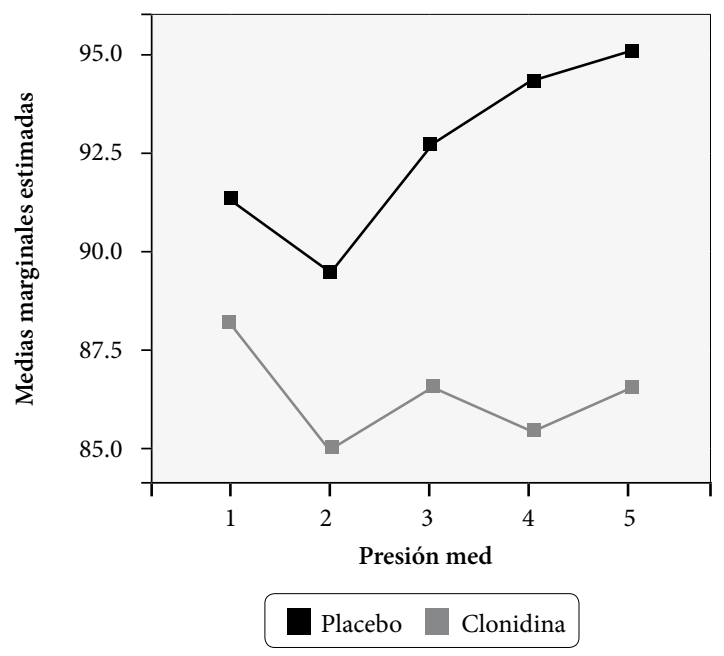

Figura 3. Tendencia de las medias marginales de la presión arterial para los grupos placebo y Clonidina. 1. Antes de administrar el medicamento; 2. Durante la anestesia; 3. En la luxación u osteotomía; 4. En la sutura; 5. Al finalizar la cirugía Fuente: elaboración propia

Los datos de la frecuencia cardíaca, la frecuencia respiratoria y la saturación de oxígeno de los pacientes de los grupos placebo y clonidina se observan en la tabla 5. El momento uno fue antes de administrar el medicamento; dos, durante la anestesia; tres, en la luxación u osteotomía; cuatro, en la sutura, y cinco, al finalizar la cirugía.

Tabla 4. Distribución de la presión arterial de los grupos placebo y clonidina

\begin{tabular}{|c|c|c|c|c|c|c|}
\hline & \multicolumn{2}{|c|}{ Sistólica } & \multicolumn{2}{|c|}{ Diastólica } & \multicolumn{2}{|c|}{ Media } \\
\hline \multirow{2}{*}{ Momento } & Placebo & Clonidina & Placebo & Clonidina & Placebo & Clonidina \\
\hline & Media \pm DS & Media \pm DS & Media \pm DS & Media \pm DS & Media \pm DS & Media \pm DS \\
\hline 1 & $123,10 \pm 15,04$ & $122,55 \pm 10,48$ & $76,60 \pm 12,27$ & $74,70 \pm 7,13$ & $91,30 \pm 12,88$ & $88,20 \pm 7,57$ \\
\hline 2 & $127 \pm 14,73$ & $122,40 \pm 13,61$ & $73,05 \pm 6,81$ & $71,10 \pm 6,20$ & $89,35 \pm 9,83$ & $84,95 \pm 8,69$ \\
\hline 3 & $128,60 \pm 16,98$ & $122,35 \pm 13,58$ & $78 \pm 10,57$ & $72,55 \pm 6,45$ & $92,70 \pm 14,94$ & $86,55 \pm 8,76$ \\
\hline 4 & $128,45 \pm 18,99$ & $121 \pm 13,84$ & $76,85 \pm 10,91$ & $73,40 \pm 6,62$ & $94,30 \pm 15,79$ & $85,40 \pm 8,71$ \\
\hline 5 & $130,55 \pm 17,13$ & $117,90 \pm 14,96$ & $78,65 \pm 11,66$ & $74,25 \pm 8,03$ & $95,10 \pm 13,98$ & $86,55 \pm 9,92$ \\
\hline
\end{tabular}

Anova de dos vías ( $\mathrm{p}>0,05)$

Fuente: elaboración propia 
Tabla 5. Distribución de la frecuencia cardíaca, la frecuencia respiratoria y la saturación de oxígeno en placebo y clonidina

\begin{tabular}{|c|c|c|c|c|c|c|}
\hline & \multicolumn{2}{|c|}{ F. cardíaca } & \multicolumn{2}{|c|}{ F. respiratoria } & \multicolumn{2}{|c|}{ Sat. de oxígeno } \\
\hline \multirow{2}{*}{ Momento } & Placebo & Clonidina & Placebo & Clonidina & Placebo & Clonidina \\
\hline & Media \pm DS & Media \pm DS & Media \pm DS & Media \pm DS & Media \pm DS & Media \pm DS \\
\hline 1 & $80,75 \pm 11,99$ & $82,60 \pm 13,76$ & $21,40 \pm 3,74$ & $22,30 \pm 3,51$ & $97 \pm 1,97$ & $96,90 \pm 1,97$ \\
\hline 2 & $81,35 \pm 12,23$ & $78,55 \pm 16,80$ & $21,70 \pm 3,52$ & $22,60 \pm 3,05$ & $98,40 \pm 1,53$ & $98,05 \pm 1,31$ \\
\hline 3 & $80,50 \pm 10,47$ & $74,80 \pm 14,35$ & $23,25 \pm 5,93$ & $21,45 \pm 6,26$ & $97,35 \pm 2,01$ & $97,60 \pm 2,03$ \\
\hline 4 & $75,55 \pm 10,62$ & $73,55 \pm 18,58$ & $24,10 \pm 6,45$ & $21,35 \pm 3,18$ & $96 \pm 2,49$ & $95,95 \pm 2,54$ \\
\hline 5 & $75,75 \pm 9,68$ & $74,60 \pm 17,30$ & $21,50 \pm 3,48$ & $19,05 \pm 3,05$ & $94,85 \pm 4,20$ & $94,50 \pm 2,66$ \\
\hline
\end{tabular}

Anova de dos vías $(\mathrm{p}>0,05)$

Fuente: elaboración propia

La frecuencia cardíaca mostró que dentro de los grupos de clonidina y placebo hubo diferencia $(\mathrm{p}=0,003)$, pero no se demostró entre los mismos $(\mathrm{p}=0,592)$; en la figura 4 se observa que en ambos grupos desciende, sin mostrar diferencia entre el placebo y la clonidina.

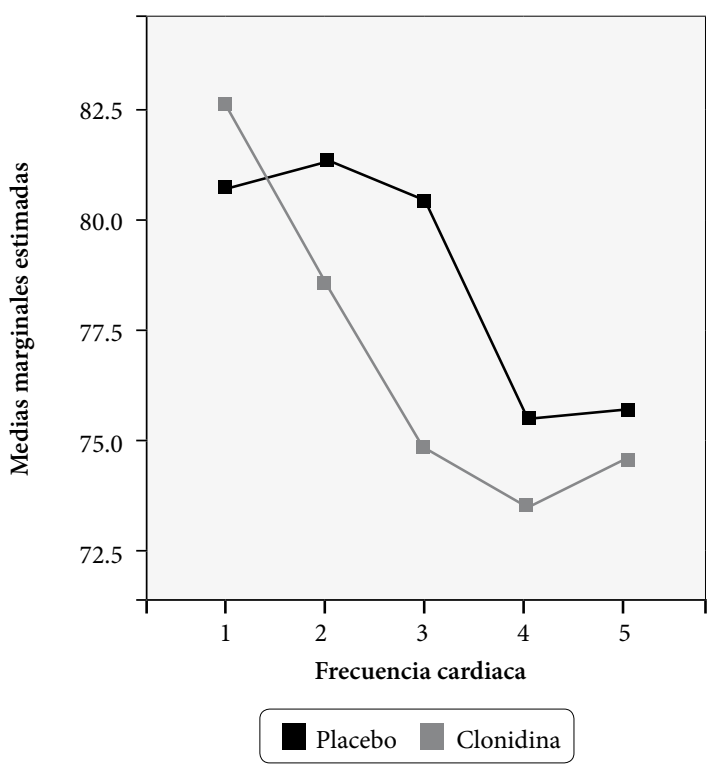

Figura 4. Medias marginales estimadas de la frecuencia cardíaca para los grupos placebo y Clonidina. 1. Antes de administrar el medicamento; 2. Durante la anestesia; 3 . En la luxación u osteotomía; 4. En la sutura; 5. Al finalizar la cirugía Fuente: elaboración propia

Al comparar la percepción del dolor entre los grupos no se encontraron diferencias estadísticamente significativas, ni durante la cirugía ni en el postoperatorio (tabla 6).
Tabla 6. Distribución del dolor intra y postoperatorio

\begin{tabular}{|c|c|c|c|c|}
\hline \multirow{2}{*}{$\begin{array}{l}\text { Escala } \\
\text { del dolor }\end{array}$} & & \multirow{2}{*}{ Valor $\mathrm{p}$} & Clonidina vs & \multirow{2}{*}{ Valor $\mathrm{p}$} \\
\hline & & & placebo & \\
\hline \multirow{2}{*}{3 horas } & Mediana & \multirow{2}{*}{0,203} & 3,00 & \multirow{2}{*}{0,148} \\
\hline & (RIQ) & & $(1,00-5,00)$ & \\
\hline \multirow{2}{*}{6 horas } & Mediana & \multirow{2}{*}{0,210} & 3,50 & \multirow{2}{*}{0,060} \\
\hline & (RIQ) & & $(1,00-6,00)$ & \\
\hline \multirow{2}{*}{24 horas } & Mediana & \multirow{2}{*}{0,127} & 1,50 & \multirow{2}{*}{0,040} \\
\hline & (RIQ) & & $(0,0-2,75)$ & \\
\hline
\end{tabular}

U de Mann Whitney $<0,05$

Fuente: elaboración propia

\section{Mann-Whitney}

En cuanto a los efectos secundarios se evidenció que náuseas, vómito, mareo y otros fueron muy poco frecuentes en los grupos del placebo y la clonidina, a diferencia de somnolencia y xerostomía, que se presentaron en mayor cantidad (tabla 7).

Tabla 7. Efectos secundarios

\begin{tabular}{|l|c|c|}
\hline \multirow{2}{*}{\multicolumn{1}{|c|}{ Variable }} & Placebo & Clonidina \\
\cline { 2 - 3 } & $\mathbf{n}(\%)$ & $\mathbf{n}(\%)$ \\
\hline Náuseas & $1(5)$ & $3(15)$ \\
\hline $3 \mathrm{~h}$ & $1(5)$ & $2(10)$ \\
\hline $6 \mathrm{~h}$ & 0 & 0 \\
\hline $12 \mathrm{~h}$ & \multicolumn{2}{|c|}{} \\
\hline Vómito & 0 & 0 \\
\hline $3 \mathrm{~h}$ & $1(5)$ & 0 \\
\hline $6 \mathrm{~h}$ & 0 & 0 \\
\hline $12 \mathrm{~h}$ &
\end{tabular}

Continúa 


\begin{tabular}{|c|c|c|}
\hline \multirow{2}{*}{ Variable } & Placebo & Clonidina \\
\hline & n (\%) & n (\%) \\
\hline \multicolumn{3}{|l|}{ Mareo } \\
\hline $3 \mathrm{~h}$ & $6(30)$ & $6(30)$ \\
\hline $6 \mathrm{~h}$ & $3(15)$ & $4(20)$ \\
\hline $12 \mathrm{~h}$ & $1(5)$ & $1(5)$ \\
\hline \multicolumn{3}{|l|}{ Somnolencia } \\
\hline $3 \mathrm{~h}$ & $4(20)$ & $11(55)$ \\
\hline $6 \mathrm{~h}$ & $8(40)$ & $17(85)$ \\
\hline $12 \mathrm{~h}$ & $1(5)$ & $4(20)$ \\
\hline \multicolumn{3}{|l|}{ Xerostomía } \\
\hline $3 \mathrm{~h}$ & $12(60)$ & $10(50)$ \\
\hline $6 \mathrm{~h}$ & $6(30)$ & $6(30)$ \\
\hline $12 \mathrm{~h}$ & 0 & $3(15)$ \\
\hline \multicolumn{3}{|l|}{ Otros (cefalea) } \\
\hline $3 \mathrm{~h}$ & $2(10)$ & 0 \\
\hline $6 \mathrm{~h}$ & 0 & $1(5)$ \\
\hline $12 \mathrm{~h}$ & $1(5)$ & $1(5)$ \\
\hline
\end{tabular}

Anova de dos vías ( $\mathrm{p}>0,05)$

Fuente: elaboración propia

Se evaluó la satisfacción de los pacientes al terminar la cirugía y la gran mayoría $(51,6 \%)$ se sintió muy satisfecha, 26 pacientes $(43,3 \%)$ estuvieron satisfechos, un paciente estuvo moderadamente satisfecho y sólo dos pacientes, uno de placebo y uno de clonidina, manifestaron insatisfacción.

\section{Discusión}

Controlar la ansiedad de los pacientes que van a ser sometidos a una cirugía dental es un reto que enfrentan diariamente los odontólogos y, en especial, los cirujanos. Para tal propósito, se parte de la relación entre los profesionales de la odontología con sus pacientes, de la manera cómo estos son abordados por el personal auxiliar, así como también de las condiciones ambientales y de las estrategias que se desencadenan con el fin de disminuir el temor que los pacientes manifiestan. Sin embargo, en muchas ocasiones esto no es suficiente, por lo cual se han utilizado otras medidas de sensibilización cognitiva [9] y un grupo de medidas farmacológicas que van desde la medicación de un ansiolítico, hasta medidas más drásticas como la sedación y la anestesia general.

El uso de fármacos está ligado a los efectos adversos que puedan desencadenar y a la seguridad e integridad física del paciente. La ansiedad por sí misma ha sido descrita como un fenómeno que afecta al individuo, que desencadena un sistema de respuesta triple que involucra lo cognitivo, lo somático y lo motor [10]. Por lo tanto, las alternativas farmacológicas deben estar dirigidas a controlar estos aspectos de la génesis de la ansiedad. Los agonistas preferenciales alfa-2 como la clonidina han sido utilizados por más de tres décadas como tratamiento de las arritmias cardíacas y la hipertensión. En este tiempo se han demostrado diferentes usos para estos fármacos, incluyendo el control del dolor y la ansiedad generada por procedimientos quirúrgicos en diferentes áreas de la salud [11-14].

En el presente estudio se encontró que los pacientes tratados tanto con la clonidina como con el placebo experimentan una disminución en los niveles de ansiedad después de recibir la intervención, con diferencias significativas con respecto a la medida inicial de la escala MDAs. Sin embargo, entre ambos grupos el comportamiento es similar, como también para los signos vitales, aunque hubo una diferencia significativa en la presión arterial media; además, al analizar las medidas marginales, estas sugieren que la tendencia de la clonidina, en cuanto a presión arterial y frecuencia cardíaca, es a mantener niveles más bajos. Los efectos secundarios se presentaron en muy pocos casos y la percepción del dolor intra y postoperatorio tampoco mostró diferencias.

Chowdhury y sus colaboradores [15] encontraron que al utilizar la clonidina en combinación con la lignocaína, comparadas con la mezcla de la lignocaína con adrenalina, en una cirugía para la extracción de terceros molares en pacientes adultos, aquellos que recibieron la clonidina mantuvieron mayor estabilidad en los signos vitales, en especial la presión arterial, con mínimos efectos secundarios. Estos resultados fueron similares a los obtenidos en el presente estudio, a pesar de que a diferencia de este, en aquel estudio la clonidina se infiltró localmente en combinación con un anestésico local [15].

Hall y sus colaboradores [12] administraron la clonidina por vía oral, comparándola con placebo, como medicación preoperatoria en pacientes adultos que serían sometidos a cirugía oral de larga duración, bajo sedación consciente; entre sus objetivos se plantearon evaluar los signos vitales y el dolor postoperatorio. 
Los resultados nuevamente confirman, al igual que en el presente estudio, que en el grupo experimental hubo mayor estabilidad en la presión arterial sistólica, diastólica y media que en el grupo de control. La frecuencia cardíaca también mostró cifras inferiores, aunque en este caso con diferencias estadísticamente significativas [16].

Cao y sus colaboradores [11], y Trevor y sus colaboradores [17] utilizaron la clonidina oral como premedicación operatoria, comparada con el Midazolam, para controlar la ansiedad y el dolor en niños, y se encontró en ambos casos una adecuada ansiolisis y una disminución de dolor, a la par que la tensión arterial se mantuvo estable y en niveles bajos, lo cual indica que en la población infantil también funciona adecuadamente la clonidina $[11,17]$.

Lizawa y sus colaboradores [18] demostraron que la clonidina, comparada con la tandospirona y un placebo, disminuyen la ansiedad preoperatoria, sin diferencias significativas, aun cuando lo hacen mediante mecanismos diferentes; este resultado es comparable al obtenido en el presente estudio [18].

El hecho de que no se encontraran diferencias en cuanto al control de ansiedad en ambos grupos, de ninguna manera desestima la utilidad de la clonidina como terapia útil en el control de la ansiedad en procedimientos quirúrgicos. Van Die y sus colaboradores [19], en un análisis posthoc de un ensayo clínico placebo controlado con dos brazos de tratamiento, para dilucidar el mecanismo por el cual actúa el placebo versus los medicamentos activos, encontraron que existen indicios de que los patrones neurofisiológicos para producir un efecto pueden ser diferentes; por lo tanto se puede dudar de un efecto la aditivo del placebo a los tratamientos con medicamentos [19].

Por otra parte, adicionalmente al control de la ansiedad, el comportamiento de los parámetros hemodinámicos en el grupo activo del presente estudio mostró ventajas en relación con el placebo; en el caso de la clonidina, en la presión arterial media se debe considerar que como antihipertensivo va a controlar la liberación de adrenalina, considerada la principal causa de la ansiedad.

En conclusión, el uso de alguna terapia, incluido un placebo, tiene un efecto benéfico en el control de la ansiedad en pacientes sometidos a cirugía oral. La clonidina estabiliza y favorece el comportamiento de los signos somáticos de la ansiedad, con mínimos efectos secundarios, incluso menos que en el placebo. En la mayoría de los casos, los pacientes manifestaron estar satisfechos con la terapia recibida. Hay que decir que dentro de las limitaciones del estudio se encuentra el hecho de que no se pudo controlar el tipo de medicación postoperatoria, lo cual hizo difícil evaluar la eficacia de los tratamientos en relación con el control del dolor postoperatorio. Futuros estudios con diferentes dosis de los medicamentos nos podrían acercar a la forma más adecuada de abordar la ansiedad y el dolor durante procedimientos quirúrgicos en odontología.

\section{Referencias}

[1] Ng SKS, Leung WK. A community study on the relationship of dental anxiety with oral health status and oral health-related quality of life. Community Dent Oral Epidemiol. 2008; 36(4): 347-56.

[2] Caycedo C, Cortés OF, Gama R, Rodríguez H, Colorado $\mathrm{P}$, Caycedo $\mathrm{M}$ et al. Ansiedad al tratamiento odontológico: Características y diferencias de género. Suma Psicológica. 2008; 15(1): 259-78.

[3] Kvale G, Berggren U, Milgrom P. Dental fear in adults: a meta-analysis of behavioral interventions. Community Dent Oral Epidemiol. 2004; 32(4): 250-64.

[4] Lahmann C, Schoen R, Henningsen P, Ronel J, Muehlbacher $\mathrm{M}$, Loew $\mathrm{T}$ et al. Brief relaxation versus music distraction in the treatment of dental anxiety: a randomized controlled clinical trial. J Am Dent Assoc. 2008; 139(3): 317-24.

[5] Kritsidima M, Newton T, Asimakopoulou K. The effects of lavender scent on dental patient anxiety levels: a cluster randomized-controlled trial. Community Dent Oral Epidemiol. 2010; 38(1): 83-7.

[6] Lee-Kim SJ, Fadavi S, Punwani I, Koerber A. Nasal versus oral midazolam sedation for pediatric dental patients. J Dent Child (Chic). 2004; 71(2): 126-30.

[7] Carreño ML, Benavente C, Acosta O, Sendín MB. Sedación y anestesia general en el paciente odontopediátrico. Gaceta dental: Ind Prof. 2008; 192: 88-111.

[8] Lago-Méndez L, Diniz-Freitas M, Senra-Rivera C, Seoane-Pesqueira G, Gándara-Rey JM, García-García A. Postoperative recovery after removal of a lower third molar: role of trait and dental anxiety. Oral Surg Oral Med Oral Pathol Oral Radiol Endod. 2009; 108(6): 855-60.

[9] Gordon D, Heimberg RG, Tellez-Merchán M, Ismail A. A Critical review of approaches to the treatment of dental anxiety in adults. J Anxiety Disord. 2013; 27(4): 365-78. 
[10] Sandín B, Chorot P, Santed MA, Jiménez P, Romero M. Ansiedad cognitiva y somática: relación con otras variables de ansiedad y psicosomáticas. Rev Psicol Gen Apl. 2002; 47(3): 313-20.

[11] Cao J, Shi X, Miao X, Xu J. Effects of premedication of midazolam or clonidine on perioperative anxiety and pain in children. Biosci Trends. 2009; 3(3): 115-8.

[12] Hall DL, Rezvan E, Tatakis DN, Walters JD. Oral clonidine pretreatment prior to venous cannulation. Anesth Prog. 2006; 53(2): 34-42.

[13] Jacob R. Entendiendo la anestesia pediátrica. $2^{\text {a }}$ Ed. [libro electrónico]. Portal Médico Moderno. 2012 [Consultado: 2 de junio del 2013]. Disponible en: http:// medicomoderno.org/entendiendo-la-anestesia-pediatrica-2a-ed-rebecca-jacob/

[14] Imai Y, Mammoto T, Murakami K, Kita T, Sakai T, Kagawa $\mathrm{K}$ et al. The effects of preanesthetic oral clonidine on total requirement of propofol for general anesthesia. J Clin Anesth. 1998; 10(8): 660-5.
[15] Chowdhury S, Singh M, Shah A. Efficacy of lignocaine with clonidine and adrenaline in minor oral surgical procedure. Contemp Clin Dent. 2012; 3(2): 227-9.

[16] Hall DL, Tatakis DN, Walters JD, Rezvan E. Oral clonidine pre-treatment and diazepam/meperidine sedation. J Dent Res. 2006; 85(9): 854-8.

[17] Trevor S, Upadya M, Sinha C, Kaur M. A comparison of midazolam and clonidine as an oral premedication in pediatric patients. Saudi J Anaesth. 2012; 6(1): 8-11.

[18] Lizawa A, Oshima T, Kasuya Y, Dohi S. Oral tandospirone and clonidine provide similar relief of preoperative anxiety. Can J Anaesth. 2004; 51(7): 668-71.

[19] Van Die MD, Bone KM, Burger HG, Teede HJ. Are we drawing the right conclusions from randomised placebo-controlled trials? A post-hoc analysis of data from a randomized controlled trial. BMC Med Res Method. 2009; 9(1): 41 . 